\title{
Some Properties from Construction of Finite Projective Planes of Order 2 and 3
}

\author{
Vira Hari Krisnawati1 ${ }^{1}$ Corina Karim² \\ Department of Mathematics FMIPA,Brawijaya University, Malang \\ Email : virahari@ub.ac.id, co mathub@ub.a.c.id
}

\begin{abstract}
In combinatorial mathematics, a Steiner system is a type of block design. Specifically, a Steiner system $S(t$, $k, v$ ) is a set of $v$ points and $k$ blocks which satisfy that every $t$-subset of $v$-set of points appear in the unique block. It is well-known that a finite projective plane is one examples of Steiner system with $t=2$, which consists of a set of points and lines together with an incidence relation between them and order 2 is the smallest order. In this paper, we observe some properties from construction of finite projective planes of order 2 and 3. Also, we analyse the intersection between two projective planes by using some characteristics of the construction and orbit of projective planes over some representative cosets from automorphism group in the appropriate symmetric group.
\end{abstract}

Keywords: automorphism group, finite projective planes, Steiner system, symmetric group.

\section{INTRODUCTION}

A finite projective plane is a geometric construction that extends the concept of a plane. It consists of a set of points and lines together with an incidence relation between them and order 2 is the smallest order. Meanwhile in combinatorial mathematics, a Steiner system is a type of block design. Specifically, a Steiner system $S(t, k, v)$ is a set of $v$ points and $k$ blocks which satisfy that every $t$-subset of $v$-set of points appear in the unique block. It is well-known that a finite projective plane is one examples of Steiner system with $t=2$, which consists of a set of points and lines together with an incidence relation between them and order 2 is the smallest order. Or we can state that a finite projective plane of order $q$, denoted by $\operatorname{PG}(2, q)$, with the lines as blocks, is an Steiner system $S\left(2, q+1, q^{2}+q+1\right)$.

Arithmetically, when a Steiner system exists, there can be no more than ${ }_{v-t} C_{k-t}$ mutually disjoint of the Steiner systems, which is called large set. In other words, a large set is a partition of the set of all $k$-subsets of $v$-set into Steiner systems $S(t, k, v)$. For finite projective planes, according to [1], there is only known a large set of projective plane of order 3 or Steiner system $S(2,4,13)[2]$, while a large set of projective plane of order 2 or Steiner system $S(2,3,7)$ do not exist and the existence of large set of projective plane of order 4 or Steiner system $S(2,5,21)$ is still unsettled problem.

Due to the fact, we observe some properties from construction of finite projective planes of order 2 and order 3 which is as a comparison. Also, we analyse the intersection between two projective planes by using some characteristics of the construction and orbit of projective planes over some representative cosets from automorphism group in the appropriate symmetric group. 
To observe and check some conditions, we use GAP software (Groups, Algorithms, and Programming).

\section{RESULTS AND DISCUSSION}

Based on Definition 1 and Theorem 1, we analyse some properties from construction of finite projective planes of order 2 and 3 by observing the characteristics of points and lines.

\section{Finite Projective Plane of Order 2}

Finite projective plane of order 2 is a projective plane whose the smallest order, or it is called Steiner system S $(2,3,7)$. Based on Definition 1 and Theorem 1, it consists of 7 points and 7 lines which there are 3 points in each line and dually every point lies on 3 lines. Also, PG(2, 2) satisfies that every 2 points of $D$ is contained in one line and dually every 2 lines of incident with one point.

\section{Construction of Finite Projective Plane of Order 2}

Let $\pi$ be a finite projective plane of order 2 or denoted by PG(2,2), the set of points $\pi$ is $D$ $=\left\{p_{i} \mid i=1,2, \mathrm{~K}, 7\right\}$, and $\mathcal{L}=\left\{l_{i} \mid i=1,2, \mathrm{~K}, 7\right\}$ is the set of lines of $\pi$. By observing these conditions, we get some characteristics which can be used to construct a finite projective plane of order 2 . Some properties of the constructions are contained in some following propositions.

Proposition 1. There are 3 important lines in $\mathcal{L}$ to generate a finite projective plane of order 2 such that other lines can be found uniquely. The 3 generator lines consist of :

2 lines from $\left\{l \in \mathcal{L} \mid p_{i} \in l\right\}$, and

1 line from $\left\{l^{\prime} \in \mathcal{L} \mid p_{i} \notin l^{\prime}\right\}$.

Proposition 2. There are 2 possibilities conditions to choose 3 lines in $\mathcal{L}$.

There exist 1 point which is contained in 3 lines. Then, the number possibilities of such 3 lines in $\mathcal{L}$ equal to 7 .

3 lines in the (i) property are generator lines which satisfy Proposition 1 . Then, the number possibilities of 3 such generator lines in $\mathcal{L}$ equal to 28 .

Proposition 3. There are 6 distinct finite projective plane of order 2 which contain one same line. And, the total number of distinct finite projective plane of order 2 is 30 .

It is well-known that the automorphism group of $\pi$ is isomorphic to Projective Special Linear Group, $\operatorname{PSL}(3,2)$. By using GAP, we can find that $\operatorname{PSL}(3,2)$ consists of some following elements which is described in Table 1.

Table 1. All Element of $\operatorname{PSL}(3,2)$ based on the Cycle

\begin{tabular}{|c|c|c|}
\hline Order & Cycle Type & The total number \\
\hline 1 & $1^{7}$ & 1 \\
\hline 2 & $1^{3} \cdot 2^{2}$ & 21 \\
\hline 3 & $1^{1} \cdot 3^{2}$ & 56 \\
\hline 4 & $1^{1} \cdot 2^{1} \cdot 4^{1}$ & 42 \\
\hline 7 & $7^{1}$ & 48 \\
\hline T o t a l & 168 \\
\hline
\end{tabular}


Corollary 1. There is unique up to isomorphic $\mathrm{PG}(2,2), \pi$ which is cyclic.

Proof. Let $G$ be the automorphism group of $\pi$. Since $|G|=2^{3} \times 3 \times 7=168$ and by deduction of Lagrange's theorem, $G$ contain a single cycle of length 7 , thus $\pi$ is cyclic. And since the index of subgroup $G$ in the symmetric group $S_{7}$,

$$
\left[S_{7}: G\right]=\frac{\left|S_{7}\right|}{|G|}=\frac{7 !}{168}=30=\#\{\pi: P G(2,2)\},
$$

there is unique up to isomorphic $\pi$.

According to Proposition 1, to construct finite projective plane $\pi$ of order 2 there are 3 important lines in $\mathcal{L}$ such that other lines can be found uniquely.

Let $X$ be the set of such 3 important lines. Based on Proposition 2, the number possibilities of $X$ equal to 28. By using GAP, we can determine the stabilizer of this type $X, \operatorname{Stab}_{G}(X) \cong S_{3}$.

1.2 The Existence of Intersection Between Two Finite Projective Planes of Order 2

Now, we analyse the number of common lines between two finite projective planes of order 2. Let $\pi$ and $\pi^{\prime}$ be two distinct PG $(2,2)$. Also, let $\mathcal{L}_{\pi}$ be the set of lines of $\pi$ and $\mathcal{L}_{\pi^{\prime}}$ be the set of lines of $\pi^{\prime}$. If $\left|\mathcal{L}_{\pi} \cap \mathcal{L}_{\pi^{\prime}}\right|=s$, it means that $\mathcal{L}_{\pi}$ and $\mathcal{L}_{\pi^{\prime}}$ intersect in $s$ lines and if $\mid \mathcal{L}_{\pi} \cap \mathcal{L}_{\pi^{\prime}}$ $\mid=0$, then $\mathcal{L}_{\pi}$ and $\mathcal{L}_{\pi^{\prime}}$ are mutually disjoint.

Proposition 4. There are no $\pi$ and $\pi^{\prime}$ such that $\left|\mathcal{L}_{\pi} \cap \mathcal{L}_{\pi^{\prime}}\right|=2$.

Proof. Supposse there is $\pi$ and $\pi^{\prime}$ such that $\left|\mathcal{L}_{\pi} \cap \mathcal{L}_{\pi^{\prime}}\right|=2$. Let $\mathcal{L}_{\pi} \cap \mathcal{L}_{\pi^{\prime}}=\left\{l_{1}, l_{2}\right\}$. Since in PG(2,2), every 2 lines incident with 1 point, let $l_{1} \cap l_{2}=\{p\}$ and every point lies on 3 lines, then we can determine $l_{3}$ which contain point $p$ uniquely. Thus, $\left|\mathcal{L}_{\pi} \cap \mathcal{L}_{\pi^{\prime}}\right|=3 \neq 2$. It is a contradiction. Hence, There is no $\pi, \pi^{\prime}$ such that $\left|\mathcal{L}_{\pi} \cap \mathcal{L}_{\pi^{\prime}}\right|=2$.

Proposition 5. There are no $\pi$ and $\pi^{\prime}$ such that $\left|\mathcal{L}_{\pi} \cap \mathcal{L}_{\pi^{\prime}}\right|=4,5$, or 6 .

Proof. Let $I=\mathcal{L}_{\pi} \cap \mathcal{L}_{\pi^{\prime}}$ and $4 \leq|I| \leq 6$. We divide the proof into two cases :

Case 1. If there are 3 generator lines in $I$ which satisfy Proposition 1 , then $\pi=\pi$ '.

Case 2. Assume $I$ do not contain generator lines based on Proposition 1. According to the construction of $\operatorname{PG}(2,2)$ and due to $|I| \geq 4$, there exist one point which incident with 3 lines in $I$. Thus, every 2 lines of such 3 lines satisfy Proposition 1 part (i). Since it still has at least one line in $I$ and based on the properties of PG(2, 2), the line satisfy Proposition 1 part (ii), so $\pi=\pi^{\prime}$. Hence, it contradicts with the asumption.

Therefore, there is no $\pi, \pi^{\prime}$ such that $\left|\mathcal{L}_{\pi} \cap \mathcal{L}_{\pi^{\prime}}\right|=4,5$, or 6 .

Based on Proposition 4 and 5, the possibilities of intersection between two $\mathrm{PG}(2,2)$ is 0 , 1,3 , or 7 lines. To examine the exsistence of this intersection, we fix $\operatorname{PG}(2,2), \pi$ and then determine the orbit of $\pi$ under some elements of symmetric group $S_{7}$.

Proposition 6. For all $x \in S_{7}$, $\mathrm{x}$ with cycle type $1^{5} .2^{1}$ then $\left|\mathrm{L}^{\pi}(\mathrm{L} \pi) \mathrm{x}\right|=3$.

Proposition 7. For all $x \in S_{7}$, $\mathrm{x}$ with cycle type $1^{4} \cdot 3^{1}$ then $\left|\mathrm{L}^{\pi} \cap(\mathrm{L} \pi) \mathrm{x}\right|=1$.

Proposition 8. For all $x \in S_{7}, x$ with cycle type $1^{3} .4^{1}$ such that there is one line in $\mathcal{L}_{\pi}$ which contains 3 points in $x$ then $\left|\mathcal{L}_{\pi} \cap\left(\mathcal{L}_{\pi}\right)^{x}\right|=0$.

Also, according to Proposition 4, Proposition 5, and the existence of common lines in Proposition 6 until Proposition 8, we can state the following theorem.

Theorem 2. $\left|\mathcal{L}_{\pi} \cap \mathcal{L}_{\pi^{\prime}}\right|=0,1,3$, or 7 , for any $\pi$ and $\pi^{\prime}$ are finite projective plane of order 2 .

Moreover based on Corollary 1 , there is unique up to isomorphism $\pi$. Thus by using the orbit of fixed $\pi$ under all elements in the right transversal of $G$, we can calculate how many numbers of $\pi^{\prime}$ such that $\left|\mathcal{L}_{\pi} \cap \mathcal{L}_{\pi^{\prime}}\right|=s$, where $s \in\{0,1,3,7\}$. 
Remark 1. Let $\pi$ be a fix PG(2,2). The number of finite projective plane $\pi^{\prime}$ of order 2 whose have the such possibilities of size common lines with $\mathcal{L}_{\pi}$, as stated in Theorem 2 is showed in the following Table 2. We can get it by using GAP.

Table 2. The Number of PG(2,2) Based on the Size of Common Lines

\begin{tabular}{|c|c|}
\hline$\left|\mathcal{L}_{\pi} \cap \mathcal{L}_{\pi^{\prime}}\right|=s$ & $\#\left\{\pi^{\prime}: \mathrm{PG}(2,2)|| \mathcal{L}_{\pi} \cap \mathcal{L}_{\pi^{\prime}} \mid=s\right\}$ \\
\hline 0 & 8 \\
\hline 1 & 14 \\
\hline 3 & 7 \\
\hline 7 & 1 \\
\hline Total & 30 \\
\hline
\end{tabular}

\section{Finite Projective Plane of Order 3}

Based on Definition 1 and Theorem 1, finite projective plane of order 3 or Steiner system $S(2,4,13)$ consists of 13 points and 13 lines which there are 4 points in each line and dually every point lies on 4 lines. Also, PG(2, 3) satisfies that every 2 points of $D$ is contained in one line and dually every 2 lines of incident with one point.

2.1. Construction of Finite Projective Plane of Order 3

Let $\pi$ be a finite projective plane of order 3 or denoted by PG $(2,3)$, the set of points $\pi$ is $D$ $=\left\{p_{i} \mid i=1,2, \mathrm{~K}, 13\right\}$, and $\mathcal{L}=\left\{l_{i} \mid i=1,2, \mathrm{~K}, 13\right\}$ is the set of lines of $\pi$. By observing these conditions, we get some characteristics which can be used to construct a finite projective plane of order 3 . Some properties of the constructions are contained in some following propositions.

Proposition 9. There are 6 important lines in $L$ to generate a finite projective plane of order 3 such that other lines can be found uniquely. Let $p_{1}, p_{2}$ be any points of $D$ and $p_{1}, p_{2} \in l_{1}$, then the 6 generator lines consist of :

3 lines from $\left\{l \in \mathcal{L} \mid p_{1} \in l\right\}$,

2 lines from $\left\{l^{\prime} \in \mathcal{L} \mid p_{2} \in l^{\prime}, p_{1} \notin l^{\prime}\right\}$, and

1 line from $\left\{l^{\prime \prime} \in \mathcal{L} \mid p_{i} \in l^{\prime \prime}, p_{1}, p_{2} \notin l^{\prime \prime}\right\}$, where $p_{i} \in l_{1} \backslash\left\{p_{1}, p_{2}\right\}$.

Proposition 10. There are 4 possibilities conditions to choose 6 lines in $\mathcal{L}$.

There exists one point which is contained in 4 lines and another point lies on one of such 4 lines and other 2 lines. Then the number possibilities of such 6 lines in $\mathcal{L}$ equal to 468 . There exist 2 points which appear in 3 lines. Then the number possibilities of such 6 lines in $\mathcal{L}$ equal to 78 . There exist 3 points which appear in 3 lines. Then the number possibilities of such 6 lines in $\mathcal{L}$ equal to 936. There exist 4 points which is contained in 3 lines. Then the number possibilities of such 6 lines in $L$ equal to 234. Thus, the number of possibilities of 6 generator lines in $\mathcal{L}$ based on Proposition 9 are part (iii) dan (iv), i.e., $936+234=1.170$.

Proposition 11. There are 20.160 distinct projective plane of order 3 that contain one same line. And, the total number of distinct finite projective plane of order 3 is 1.108.800.

It is well-known that the automorphism group of $\pi$ is isomorphic to Projective Special Linear Group, $\operatorname{PSL}(3,3)$. By using GAP, we can find that $\operatorname{PSL}(3,3)$ consists of some following elements which is described in Table 3.

Table 3. All Element of $\operatorname{PSL}(3,3)$ based on the Cycle

\begin{tabular}{|l|l|l|}
\hline Order & Cycle Type & The total number \\
\hline 1 & $1^{13}$ & 1 \\
\hline 2 & $1^{5} \cdot 2^{4}$ & 117 \\
\hline \multirow{2}{*}{3} & $1^{4} \cdot 3^{3}$ & 104 \\
\cline { 2 - 3 } & $1^{1} \cdot 3^{4}$ & 624 \\
\hline
\end{tabular}




\begin{tabular}{|l|l|l|}
\hline 4 & $1^{1} \cdot 2^{2} \cdot 4^{2}$ & 702 \\
\hline 6 & $1^{2} \cdot 2^{1} \cdot 3^{1} \cdot 6^{1}$ & 936 \\
\hline 8 & $1^{1} \cdot 4^{1} \cdot 8^{1}$ & 1404 \\
\hline 13 & $13^{1}$ & 1728 \\
\hline T o t a l & & 5616 \\
\hline
\end{tabular}

Corollary 2. There is unique up to isomorphic $\mathrm{PG}(2,3), \pi$ which is cyclic.

Proof. Let $G$ be the automorphism group of $\pi$. Since $|G|=2^{4} \times 3^{3} \times 13=5.616$ and by deduction of Lagrange's theorem, $G$ contain a single cycle of length 13 , thus $\pi$ is cyclic. And since the index of subgroup $G$ in the symmetric group $S_{13}$,

$$
\left[S_{13}: G\right]=\frac{\left|S_{13}\right|}{|G|}=\frac{13 !}{5.616}=1.108 .800=\#\{\pi: P G(2,3)\},
$$

there is unique up to isomorphic $\pi$.

According to Proposition 9, to construct finite projective plane $\pi$ of order 3 there are 6 important lines in $\mathcal{L}$ such that other lines can be found uniquely.

Let $X$ be the set of such 6 important lines. Based on Proposition 10, we can state that there are 2 types of $X$ :

There are 3 points that incident with 3 lines in $X$. The number possibilities of such $X$ equal to 936 . By using GAP, we can determine the stabilizer of this type $X, \operatorname{Stab}_{G}(X) \cong S_{3}$.

There are 4 points that incident with 3 lines in $X$. The number possibilities of such $X$ equal to 234 . By using GAP, we can determine the stabilizer of this type $X, \operatorname{Stab}_{G}(X) \cong S_{4}$.

2.2 The Existence of Intersection Between Two Finite Projective Planes of Order 3

In this section, we analyse the number of common lines between two finite projective planes of order 3. Let $\pi$ and $\pi^{\prime}$ be two distinct PG(2,3). Also, let $L_{\pi}$ be the set of lines of $\pi$ and $\mathcal{L}_{\pi^{\prime}}$ be the set of lines of $\pi^{\prime}$. If $\left|\mathcal{L}_{\pi} \cap \mathcal{L}_{\pi^{\prime}}\right|=s$, it means that $\mathcal{L}_{\pi}$ and $\mathcal{L}_{\pi^{\prime}}$ intersect in $s$ lines and if $\left|\mathcal{L}_{\pi} \cap \mathcal{L}_{\pi^{\prime}}\right|=0$, then $\mathcal{L}_{\pi}$ and $\mathcal{L}_{\pi^{\prime}}$ are mutually disjoint.

Proposition 12. There are no $\pi$ and $\pi^{\prime}$ such that $\left|\mathrm{L}^{\cap} \cap \mathrm{L} \pi^{\prime}\right|=6$.

Proposition 13. There are no $\pi$ and $\pi^{\prime}$ such that $\left|\mathcal{L}_{\pi} \cap \mathcal{L}_{\pi^{\prime}}\right|=8,9,10,11$, or 12 .

Based on Proposition 12 and 13, the possibilities of intersection between two PG(2, 3) is $0,1,2,3,4,5,7$, or 13 lines. To observe the exsistence of this intersection, we fix PG(2, 3), $\pi$ and then determine the orbit of $\pi$ under some elements of symmetric group $S_{13}$.

Proposition 14. For all $x \in S_{13}, x$ with cycle type $1^{11} .2^{1}$ then $\left|\mathcal{L}_{\pi} \cap\left(\mathcal{L}_{\pi}\right)^{x}\right|=7$.

Proof. From the cycle type of $x, x$ fixes 11 points and let $x=\left(p_{1}, p_{2}\right)$. Since in $\pi$ every 2 points appear in the one line, so there is unique $l \in \mathcal{L}$ such that $\left\{p_{1}, p_{2}\right\} \subset l$ then $(l)^{x}=l \in\left(\mathcal{L}_{\pi}\right)^{x}$. And, since every point lies on 4 lines,

$$
\#\left\{l^{\prime} \in \mathcal{L}_{\pi} \mid p_{1} \in l^{\prime}, p_{2} \notin l^{\prime}\right\}=\#\left\{l^{\prime \prime} \in \mathcal{L}_{\pi} \mid p_{1} \notin l^{\prime \prime}, p_{2} \in l^{\prime \prime}\right\}=3,
$$

then such 6 lines satisfy $\left(l^{\prime}\right)^{x} \notin \mathcal{L}_{\pi}$ because $\left|l^{\prime} \cap\left(l^{\prime}\right)^{x}\right|=3$, so do the line $l^{\prime \prime}$. Also, the other 6 lines are fixed by $x$, since they just consist of fix points. Thus, $\left|\mathcal{L}_{\pi} \cap\left(\mathcal{L}_{\pi}\right)^{x}\right|=7$.

Proposition 15. For all $x \in S_{13}$, $\mathrm{x}$ with cycle type $1^{9} \cdot 2^{2}$ then $\left|\mathrm{L} \pi^{\cap}(\mathrm{L} \pi) \mathrm{x}\right|=1,3$, or 5 .

Proposition 16. For all $x \in S_{13}$, $\mathrm{x}$ with cycle type $1^{10} \cdot 3^{1}$ then $\left|\mathrm{L}^{\cap}\left(\mathrm{L}^{\pi}\right) \mathrm{x}\right|=4$.

Proposition 17. For all $x \in S_{13}$, $\mathrm{x}$ with cycle type $1^{9} \cdot 4^{1}$ then $\left|\mathrm{L} \pi^{\cap}(\mathrm{L} \pi) \mathrm{x}\right|=1,2$, or 3 .

Proposition 18. For all $x \in S_{13}, x$ with cycle type $1^{8} .5^{1}$ then $\left|\mathcal{L}_{\pi} \cap\left(\mathcal{L}_{\pi}\right)^{x}\right|=0,1$, or 2 .

According to Proposition 12 and Proposition 13, also the existence of common lines in Proposition 14 until Proposition 18, we can state the following theorem. 
Theorem 3. $\left|\mathcal{L}_{\pi} \cap \mathcal{L}_{\pi^{\prime}}\right|=0,1,2,3,4,5,7$ or 13 , for all $\pi$ and $\pi^{\prime}$ are finite projective plane of order 3.

Based on Corollary 2, there is unique up to isomorphism $\pi$. Thus by using the orbit of fixed $\pi$ under all elements in the right transversal of $G$, we can calculate how many numbers of $\pi^{\prime}$ such that $\left|\mathcal{L}_{\pi} \cap \mathcal{L}_{\pi^{\prime}}\right|=s$, where $s \in\{0,1,2,3,4,5,7,13\}$.

Remark 2. Let $\pi$ be a fix PG(2,3). The number of finite projective plane $\pi^{\prime}$ of order 3 whose have the such possibilities of size common lines with $L_{\pi}$, as stated in Theorem 3 is showed in the following Table 4 . We can get it by using GAP.

Table 4. The Number of PG $(2,3)$ Based on the Size of Common Lines

\begin{tabular}{|c|l|}
\hline $\mid \mathcal{L}_{\pi \cap \mathcal{L}_{\pi^{\prime}} \mid=s}$ & $\#\left\{\pi^{\prime}: \mathrm{PG}(2,3)|| \mathcal{L}_{\pi} \cap \mathcal{L}_{\pi^{\prime}} \mid=s\right\}$ \\
\hline 0 & 891.972 \\
\hline 1 & 179.712 \\
\hline 2 & 30.888 \\
\hline 3 & 4.914 \\
\hline 4 & 884 \\
\hline 5 & 351 \\
\hline 7 & 78 \\
\hline 13 & 1 \\
\hline Total & 1.108 .800 \\
\hline
\end{tabular}

\section{CONCLUSION}

A finite projective plane is a geometric construction that extends the concept of a plane. It consists of a set of points and lines together with an incidence relation between them and order 2 is the smallest order. In this paper we observe some properties from construction of finite projective planes of order 2 and order 3 which is as a comparison. Also, we analyse the intersection between two projective planes by using some characteristics of the construction and orbit of projective planes over some representative cosets from automorphism group in the appropriate symmetric group.

Generally, there are some analysis results of finite projective planes of order 2, PG(2, 2) and order $3, \mathrm{PG}(2,3)$. The construction of $\mathrm{PG}(2,2)$ depends on 3 generator lines satisfying specific conditions such that 4 other lines can be determined uniquely, which are stated in Proposition 1. While the construction of $\mathrm{PG}(2,3)$ depends on 6 generator lines satisfying certain conditions such that 7 other lines can be found uniquely, which are stated in the Proposition 9. Furthermore, the existence of intersection between two $\mathrm{PG}(2,2)$ is $0,1,3$, or 7 common lines as listed in the Theorem 2 and the number of distinct PG $(2,2)$ which have same intersection described in Table 2. Meanwhile, the existence of intersection between two $P G(2,3)$ is $0,1,2,3,4,5$ or 13 common lines as listed in the Theorem 3 and the number of distinct $\mathrm{PG}(2,3)$ which have same intersection described in Table 4.

Based on the results, there are significant differencs between the characteristics of finite projective plane of order 2 and 3. Therefore, we need to analyse further for observing the existence of the large set. 


\section{REFERENCES}

[1] S. S. Magliveras, "Large sets of t-designs from groups," Math. Slovaca 59, pp. 19-38, 2009.

[2] L. G. Chouinard II, "Partitions of the 4 Subsets of a 13-Set Into Disjoint Projective Planes, Discrete Mathematics," 1983. 